\title{
Asymptotic rejection of sinusoidal disturbances based voltage balance control in back-to-back power converters
}

\author{
Francisco Umbría*,†, Francisco Gordillo, Francisco Salas, Javier Aracil and \\ Fabio Gómez-Estern \\ Department of Systems Engineering and Automation, University of Seville, 41092 Seville, Spain
}

\begin{abstract}
SUMMARY
This paper addresses the imbalance problem of the dc-link capacitor voltages in the three-level diodeclamped back-to-back power converter. In order to cope with it, a mathematical analysis of the capacitor voltage difference dynamics, based on a continuous model of the converter, is first carried out. It leads to an approximated model which contains explicitly several sinusoidal functions of time. In view of this result, the voltage imbalance phenomenon can be addressed as an output regulation problem, considering the sinusoidal functions as exogenous disturbances. Thus, a novel approach to deal with the mentioned problem in the backto-back converter is presented. Then, the particular features of the disturbances are used to design several controllers. They all follow an asymptotic disturbance rejection approach. In this way, the estimations of the disturbances are used to apply a control law that cancels them while regulating the capacitor voltage balance as well. Finally, the performance of the proposed control laws is evaluated, presenting the simulation results obtained when the different controllers are implemented. Copyright (c) 2014 John Wiley \& Sons, Ltd.
\end{abstract}

Received ...

KEY WORDS: back-to-back power converter; diode-clamped converter (DCC); voltage balancing; disturbance rejection; internal model principle; state observer; adaptive algorithm

\section{INTRODUCTION}

In the field of power conversion, back-to-back converter topology plays a fundamental role, that has found widespread applications such as train traction systems, automotive applications, energy generation and conversion, among others, in various industrial sectors [1]. In particular, regenerative conveyors and variable speed drives [2-4], for conversion from a three-phase source to a three-phase load with an arbitrary frequency and amplitude, and, especially, wind power generators [5,6], due to the recently increasing influence of the renewable energies [7,8], have been under research and development over the last years.

In respect of the back-to-back configuration, it consists of a connection of two power converters. The left-hand side converter generally works as a rectifier for the grid interface, and the right-hand side one as an inverter for the load, as shown in Fig. 1. Both converters are able to switch their operation functions and to connect asynchronous systems, allowing an indirect ac-ac conversion $[9,10]$, also referred to as ac-dc-ac conversion, including energy storage elements in the dc-link. These elements provide a decoupling of both converter stages for control purposes [11], as well as energy buffering when the instantaneous powers in the rectifier and inverter are unbalanced. Besides, the back-to-back converter presents other remarkable features such as good dynamic response, the

\footnotetext{
*Correspondence to: Francisco Umbría, Departamento de Ingeniería de Sistemas y Automática, Universidad de Sevilla, Camino de los Descubrimientos s/n, 41092 Sevilla, España.

†E-mail: fumbria@us.es
} 


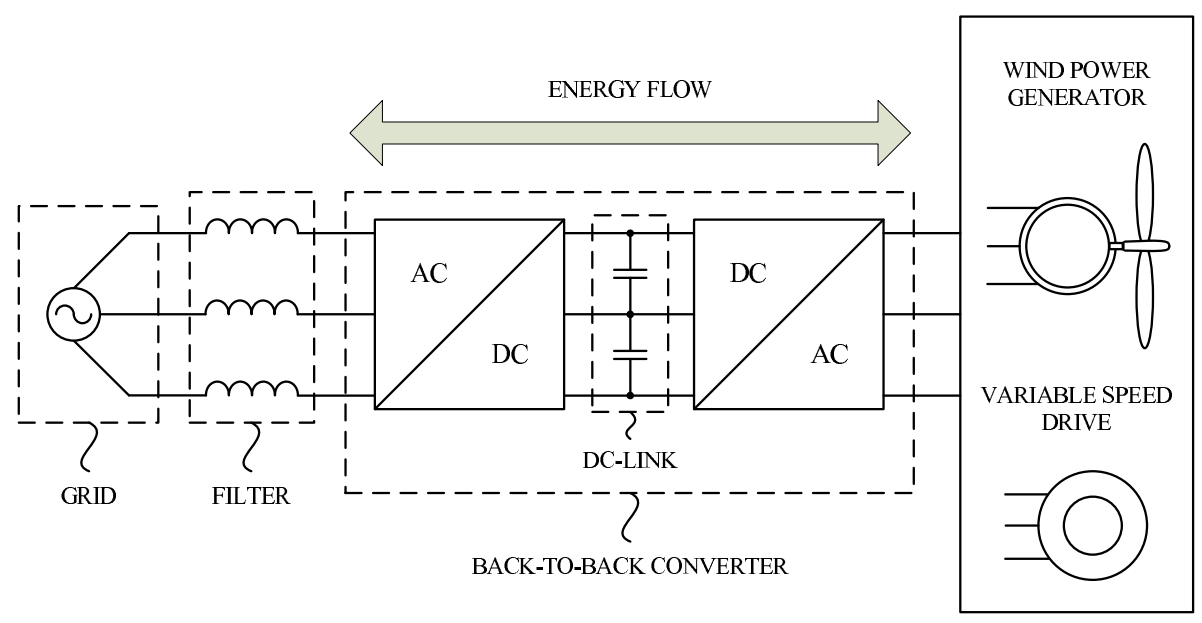

Figure 1. Three-phase three-level diode-clamped back-to-back power converter used as a grid interface for applications such as wind power generators or variable speed drives.

same hardware is required for both converters, high power factor and bidirectional power flow. In other words, they absorb energy from the grid or transfer it to the grid, meaning that the active power flow direction can be reversed at any instant due to the four-quadrant capability of the setup.

Among the different back-to-back configurations, the setup based on the three-level diodeclamped converter (DCC), also known as neutral-point-clamped converter [12], is one of the most attractive converter topologies [13,14]. It adds the typical advantages of the multilevel topology, compared with the conventional two-level converter topology, to those provided by the back-toback connection. In this way, it leads to an increase of the output voltage magnitude, lower switching frequency, reduction of the total harmonic distortion, better output voltage spectrum, robustness and higher efficiency [15]. Nevertheless, there exists the issue of the voltage imbalance of the dc-link capacitors, which is a relevant and well-known drawback of the DCC.

Concerning the difficulty of balancing of the dc-link capacitor voltages, multilevel DCCs with a large number of levels present some theoretical and practical limits related to the modulation index and the power factor of the load [16], so a proper voltage balancing is not possible in all the operating conditions [17]. Focusing on the back-to-back converter, although the particular connection of the rectifier and the inverter might yield a natural compensation of the voltage unbalance, this configuration is only able to achieve an acceptable voltage balancing including a suitable controller or modulation strategy [18-20]. Thus, the most popular method to mitigate the voltage imbalance is the use of modulation strategies based on the exploitation of the redundant switching states of the converter [21,22]. That is, those distinct positions of the converter switches that generate the same output voltage but they cause different effects on the capacitor voltages.

This paper addresses the voltage imbalance phenomenon in the three-level diode-clamped backto-back converter. In order to cope with it, an analysis of the dc-link capacitor voltage difference dynamics is carried out in this paper. The analysis is based on a continuous model of the converter and, considering a singular perturbation approach [23], yields a particular model for the voltage difference dynamics that contains explicitly several sinusoidal functions of time. This important fact is the key of the work presented here since it leads to relate the voltage imbalance problem to a problem of periodic disturbance cancellation and regulation of the output of the system. In this way, this new problem statement, which has been widely studied in the literature and applied to many other industrial system, is the first contribution of the current paper. The mathematical analysis derived enables the use of classical control theory to design the controller. Thereby, it provides an alternative approach to deal with the problem, compared with the strategies usually considered and previously mentioned [18-22].

One of the most important problems in control theory is that of controlling a fixed plan in order to retain closed-loop stability and output regulation in the presence of disturbances produced by 


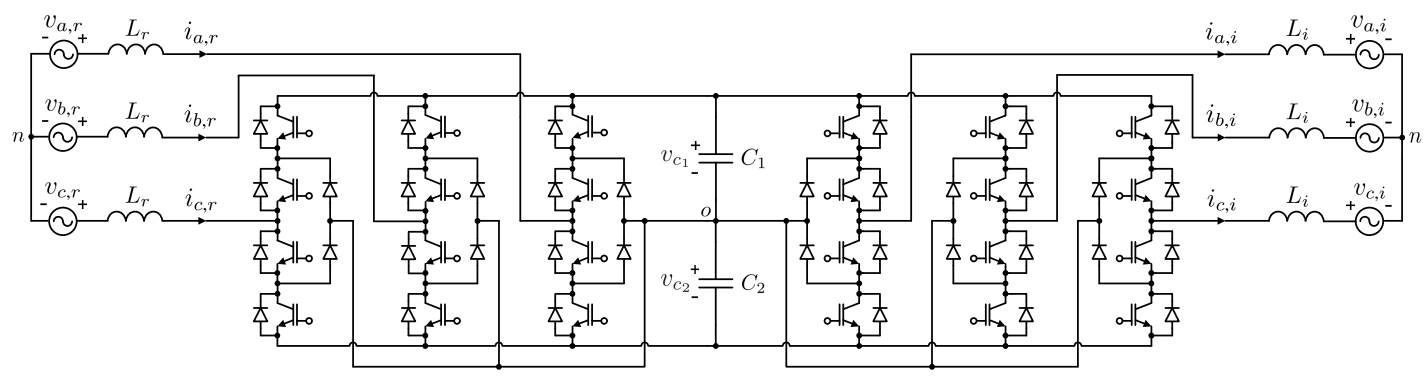

Figure 2. Three-phase three-level diode-clamped back-to-back power converter.

some external generator. The problem has been first treated for linear systems [24-26], showing that any regulator which solves the matter at hand incorporates in the feedback a reduplicated model of the dynamic structure of the disturbance which must be tracked and/or rejected. This property is commonly known as the internal model principle [25, 26]. Incorporating the earlier results established in the linear setting, the theory was then extended to the nonlinear case. The most classical formulation of the problem for this case was presented by Isidori and Byrnes in [27]. Still today, significant effort is being made in this research field [28-30].

Regarding the topic of identifying and asymptotically rejecting periodic disturbances, huge progresses have been reported [31-33]. Both the case when the disturbance frequency is known as well as the case when it is an unknown parameter have been studied. Even the varying frequency disturbance scenario, typical in applications such as rolling machines and magnetic tape drives, has been also discussed [34,35]. Thus, methods related to adaptive control [36, 37], repetitive control [38,39], backstepping [40], among others, have been used providing solid results.

Based on the analysis of the converter dynamics presented in the first part of this paper, some control schemes are designed to achieve the voltage balancing of the dc-link capacitors of the backto-back converter. In this way, the design of the controllers applying well-known techniques to reject sinusoidal disturbances is the second contribution of this work. The authors have worked previously with control techniques based on disturbance rejection in other converter topologies [41,42], where the circuit studied was the neutral-point-clamped rectifier. In particular, in [41] an analysis of the voltage difference dynamics was also formulated but leading to an expression which contains only one sinusoidal function. In the current paper, the ideas presented in [41,42] are adopted and extended to the multiple frequency approach of the back-to-back topology.

The remainder of this paper is organized as follows. In Section 2, the converter model is described, presenting the control objectives. Afterwards, the dynamics of the capacitor voltage difference are analyzed in detail in Section 3. Considering the previous analysis, Section 4 states the problem of disturbance rejection in the converter topology of this paper and proposes several control methods to guarantee the correct behavior of the capacitor voltages. Then, the admissible implementations of the proposed control laws are discussed in Section 5. The simulation results obtained are presented in Section 6. Finally, some conclusions are drawn in Section 7.

\section{MODEL OF THE BACK-TO-BACK CONVERTER AND CONTROL OBJECTIVES}

Fig. 2 illustrates a schematic diagram of the three-phase three-level diode-clamped back-to-back power converter, which is the setup considered in this paper. The dc-link is composed of capacitors $C_{1}$ and $C_{2}$, both of identical capacitance $C$, being their respective voltages $v_{c_{1}}$ and $v_{c_{2}}$. As mentioned before, both left-hand side and right-hand side converters are able to switch their operation modes. However, in the following, the left-hand side converter is named rectifier, and the right-hand side one is named inverter, in order to distinguish one from the other, as well as to simplify the notation. Both converters are connected to a corresponding ac system through the inductors $L_{r}$ and $L_{i}$. The whole system represents a general back-to-back converter. In other words, it is not only focused on a particular application of this configuration, but it includes the all purpose 
Table I. Variables of the system in $\alpha \beta \gamma$ coordinates.

\begin{tabular}{ll}
\hline Variable & Description \\
\hline $\boldsymbol{i}_{\boldsymbol{\alpha} \boldsymbol{\beta}, \boldsymbol{r}}=\left[\begin{array}{ll}i_{\alpha, r} & i_{\beta, r}\end{array}\right]^{T}$ & Rectifier phase current vector \\
$\boldsymbol{i}_{\boldsymbol{\alpha} \boldsymbol{\beta}, \boldsymbol{i}}=\left[\begin{array}{ll}i_{\alpha, i} & i_{\beta, i}\end{array}\right]^{T}$ & Inverter phase current vector \\
$\boldsymbol{v}_{\boldsymbol{\alpha} \boldsymbol{\beta}, \boldsymbol{r}}=\left[\begin{array}{ll}v_{\alpha, r} & v_{\beta, r}\end{array}\right]^{T}$ & Rectifier phase voltage vector \\
$\boldsymbol{v}_{\boldsymbol{\alpha} \boldsymbol{\beta}, \boldsymbol{i}}=\left[\begin{array}{lll}v_{\alpha, i} & v_{\beta, i}\end{array}\right]^{T}$ & Inverter phase voltage vector \\
$\boldsymbol{\delta}_{\boldsymbol{\alpha} \boldsymbol{\beta} \boldsymbol{\gamma}, \boldsymbol{r}}=\left[\begin{array}{lll}\delta_{\alpha, r} & \delta_{\beta, r} & \delta_{\gamma, r}\end{array}\right]^{T}$ & Rectifier control vector \\
$\boldsymbol{\delta}_{\boldsymbol{\alpha} \boldsymbol{\beta} \boldsymbol{\gamma}, \boldsymbol{i}}=\left[\begin{array}{lll}\delta_{\alpha, i} & \delta_{\beta, i} & \delta_{\gamma, i}\end{array}\right]^{T}$ & Inverter control vector \\
\hline
\end{tabular}

interconnection of two ac power systems with different frequencies, where the instantaneous power flows from one ac system to another.

The variables of the system in $a b c$ coordinates are, on one hand, the phase currents and phase voltages of the rectifier, denoted by $i_{a, r}, i_{b, r}, i_{c, r}$ and $v_{a, r}, v_{b, r}, v_{c, r}$, respectively. On the other hand, considering the inverter, the phase currents $i_{a, i}, i_{b, i}, i_{c, i}$, and the phase voltages, given in this case by $v_{a, i}, v_{b, i}$ and $v_{c, i}$. In addition, the gating signals that regulate the positions of the switches of both converters are defined by $\delta_{a, r}^{*}, \delta_{b, r}^{*}$ and $\delta_{c, r}^{*}$ in the rectifier, and by $\delta_{a, i}^{*}, \delta_{b, i}^{*}$ and $\delta_{c, i}^{*}$ in the inverter.

Among the large variety of converter models such as, e.g., time-varying and time-invariant averaged models, detailed switching models and small signal models [43], the continuous model of the back-to-back converter presented in [44] is adopted in this paper. The model is based on a direct average of the characteristics and waveforms associated with each of the components of the converters, providing a model valid all over the range of values of the control inputs. Thereby, the model obtained describes, in $\alpha \beta \gamma$ orthogonal coordinates, using the Clarke-Concordia Transform, the dynamics of the phase currents and of the total dc-link voltage [44], including the dc-link capacitor voltage difference dynamics, as well. Particularly, these last dynamics are given by

$$
\begin{aligned}
C \frac{d v_{d}}{d t}= & \frac{\boldsymbol{\delta}_{\boldsymbol{\alpha} \boldsymbol{\beta}, \boldsymbol{r}}^{\boldsymbol{T}} \boldsymbol{i}_{\boldsymbol{\alpha} \boldsymbol{\beta}, \boldsymbol{r}}}{\sqrt{3}} \delta_{\gamma, r}+\left[\frac{\delta_{\alpha, r}^{2}-\delta_{\beta, r}^{2}}{2 \sqrt{6}}-\frac{\delta_{\alpha, r} \delta_{\beta, r}}{\sqrt{6}}\right] \boldsymbol{i}_{\boldsymbol{\alpha} \boldsymbol{\beta}, \boldsymbol{r}} \\
& -\frac{\boldsymbol{\delta}_{\boldsymbol{\alpha} \boldsymbol{\beta}, \boldsymbol{i}}^{\boldsymbol{T}} \boldsymbol{i}_{\boldsymbol{\alpha} \boldsymbol{\beta}, \boldsymbol{i}}}{\sqrt{3}} \delta_{\gamma, i}-\left[\frac{\delta_{\alpha, i}^{2}-\delta_{\beta, i}^{2}}{2 \sqrt{6}}-\frac{\delta_{\alpha, i} \delta_{\beta, i}}{\sqrt{6}}\right] \boldsymbol{i}_{\boldsymbol{\alpha} \boldsymbol{\beta}, i},
\end{aligned}
$$

where $v_{d}$ is the difference between the upper and lower dc-link capacitor voltages, that is,

$$
v_{d}=\frac{v_{c_{1}}-v_{c_{2}}}{2} \text {. }
$$

Notice that the system variables have been transformed into $\alpha \beta \gamma$ coordinates, yielding the vectors summarized in Table I. It is assumed that the gating signals can be replaced by their averaged values in a switching period, defining in this way the control vectors $\delta_{\alpha \beta \gamma, r}$ and $\delta_{\alpha \beta \gamma, i}$. They are the control inputs of the model and are carried out as an indirect control through a modulator. The components $\alpha \beta$ of these control vectors are also represented together in (1) by

$$
\begin{aligned}
\boldsymbol{\delta}_{\boldsymbol{\alpha} \boldsymbol{\beta}, \boldsymbol{r}} & =\left[\begin{array}{ll}
\delta_{\alpha, r} & \delta_{\beta, i}
\end{array}\right]^{T} \\
\boldsymbol{\delta}_{\boldsymbol{\alpha} \boldsymbol{\beta}, \boldsymbol{i}} & =\left[\begin{array}{ll}
\delta_{\alpha, i} & \delta_{\beta, i}
\end{array}\right]^{T} .
\end{aligned}
$$

Moreover, (1) includes the current vectors $i_{\alpha \beta, r}$ and $i_{\alpha \beta, i}$, meaning that the capacitor voltage difference dynamics are coupled with the current ones.

\subsection{Control objectives}

As pointed out in the introduction, this paper addresses the imbalance problem of the voltages of the dc-link capacitors. The voltage difference $v_{d}$ should be controlled to avoid the presence of unbalance 
in the system, so it should be kept close to zero

$$
v_{d} \simeq 0
$$

providing closed-loop stability as well. It is worth stressing that it is not aimed for $v_{d}(t) \rightarrow 0$ as $t \rightarrow \infty$ for all possible initial conditions of the closed-loop state variables. Consequently, the requirements of the classical output regulation problem [24,27] are smoothed. Besides, at this point of the paper, the disturbances in the system have not been considered yet.

The analysis and control methods presented in the next sections have been worked out to keep balanced the dc-link capacitor voltages. Nevertheless, other requirements of the system such as, e.g., desired instantaneous active power, total dc-link voltage and power factor at both ac sides should be also fulfilled. Hence, it is necessary to design some other controllers to satisfy these other aims. Thus, the other variables to control are the rectifier instantaneous active and reactive powers denoted by $p_{r}$ and $q_{r}$, and in the inverter by $p_{i}$ and $q_{i}$, being their references described, respectively, by $p_{r}^{*}$, $q_{r}^{*}, p_{i}^{*}$ and $q_{i}^{*}$, and the total dc-link voltage defined as

$$
v_{d c}=v_{c_{1}}+v_{c_{2}} \text {. }
$$

In the following section it is assumed that there exist certain controllers to deal with the regulation of the instantaneous powers as well as with the total dc-link voltage. Specifically, the control vectors (3) and (4) are used by the power controller to this end, remaining control inputs $\delta_{\gamma, r}$ and $\delta_{\gamma, i}$ as the degrees of freedom for coping with the voltage imbalance problem.

\section{ANALYSIS OF THE DC-LINK CAPACITOR VOLTAGE DIFFERENCE DYNAMICS}

Since current and dc-link capacitor voltage difference dynamics in the back-to-back converter are tightly coupled, the design of a controller to balance the capacitor voltages is not an easy task. This section is devoted to study in detail the voltage difference equation in order to provide a better understanding of its behavior, as well as some remarkable results that are the basis of the proposed control methods in this paper. The analysis, worked out from a mathematical point of view, is carried out considering some essential assumptions related to a time-scale approach, that are stated next.

\subsection{Time-scale assumptions}

\section{Assumption 1}

The instantaneous power dynamics are faster than the dc-link capacitor voltage difference dynamics.

Under the assumption of different velocities of the power and voltage dynamics [45], and in order to study (1), it leads to suppose that the instantaneous powers have been regulated around their references. Therefore, applying $\delta_{\alpha \beta, r}$ and $\delta_{\alpha \beta, i}$ in an appropriate control scheme, the instantaneous powers at both rectifier and inverter sides are approximated by

$$
\begin{aligned}
p_{r} & \simeq p_{r}^{*} \\
q_{r} & \simeq q_{r}^{*} \\
p_{i} & \simeq p_{i}^{*} \\
q_{i} & \simeq q_{i}^{*} .
\end{aligned}
$$

Assumption 2

The total dc-link voltage and the rectifier instantaneous active power reference dynamics are slower than the dc-link capacitor voltage difference dynamics.

Concerning this second assumption, which is related to the design of the parameters of the voltage balancing methods, it leads to assume that the total dc-link voltage as well as the rectifier instantaneous active power reference are treated as constants in the analysis of (1). 
Regarding the rectifier power reference $p_{r}^{*}$, it is assigned to control the total dc-link voltage, whose dynamics considering Assumption 1 are given by

$$
C \frac{d}{d t}\left(\frac{v_{d c}^{2}}{2}\right) \simeq p_{r}^{*}-p_{i}^{*} .
$$

It is worth mentioning that, in the back-to-back topology, the rectifier and inverter are usually controlled individually, so one of them should be devoted to regulate the total dc-link voltage regardless of the operating state of the another. Hence, whereas the power references $q_{r}^{*}, p_{i}^{*}$ and $q_{i}^{*}$ are fixed parameters, the dynamics of the rectifier power reference $p_{r}^{*}$ are defined to drive the total dc-link voltage towards its reference $v_{d c}^{*}$.

\subsection{Final expression of the voltage difference dynamics}

Considering the time-scale assumptions and following the procedure to analyze (1) given in Appendix A, it results in the final expression of the dynamics of the voltage difference between the dc-link capacitors defined by

$$
C \frac{d v_{d}}{d t}=k_{r} \delta_{\gamma, r}-k_{i} \delta_{\gamma, i}+\phi_{r}+\phi_{i}
$$

Due to Assumption 2, $k_{r}$ and $k_{i}$ are the constants described by

$$
\begin{aligned}
k_{r} & =\frac{2 p_{r}^{*}}{\sqrt{3} v_{d c}} \\
k_{i} & =\frac{2 p_{i}^{*}}{\sqrt{3} v_{d c}} .
\end{aligned}
$$

and $\phi_{r}$ and $\phi_{i}$ are the sinusoidal functions given by

$$
\begin{aligned}
& \phi_{r}=\mu_{1, r} \sin \left(3 \omega_{r} t+3 \theta_{r}+\arctan \left(\mu_{2, r}\right)\right) \\
& \phi_{i}=\mu_{1, i} \sin \left(3 \omega_{i} t+3 \theta_{i}+\arctan \left(\mu_{2, i}\right)\right) .
\end{aligned}
$$

Parameters $\mu_{1, r}, \mu_{2, r}, \mu_{1, i}$ and $\mu_{2, i}$ are constant values defined in Appendix A. Notice that the frequencies of the sinusoidal functions correspond with three times the frequencies of the rectifier and inverter side phase voltages (77)-(80), denoted by $\omega_{r}$ and $\omega_{i}$, respectively.

Summarizing, this section has simplified the initial highly complicated expression of the dc-link capacitor voltage difference dynamics (1), leading to a simpler equation (12), under some particular conditions. Besides, (12) is a linear function of control inputs $\delta_{\gamma, r}$ and $\delta_{\gamma, i}$, containing also explicitly two additional terms which take the form of sinusoidal functions. The special features of $\phi_{r}$ and $\phi_{i}$, which are regarded in the following as external disturbances, are used to propose several methods to design the voltage balance controllers.

\section{PROBLEM STATEMENT AND PROPOSED VOLTAGE BALANCING METHODS}

Due to the appearance of the disturbances in the capacitor voltage difference dynamics, it leads to relate the voltage imbalance problem to a problem of periodic disturbance rejection. In this way, the purpose of the controllers to design is now twofold. The first goal, as pointed out in Section 2.1, is to regulate the dc-link capacitor voltage difference $v_{d}$ which should remain close to its reference given by $v_{d}^{*}$, providing closed-loop stability. This reference is set to zero to keep balanced the capacitor voltages. The second goal is the asymptotic cancellation of the sinusoidal disturbances, that is, the asymptotic cancellation of the disturbances $\phi_{r}$ and $\phi_{i}$ defined in the previous section. In this way, to achieve a proper output regulation, suitable models of both disturbances should be included in the controller, as stated in $[25,26]$. 


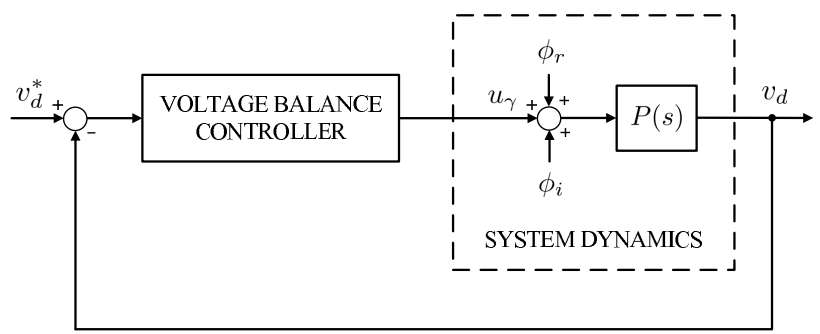

Figure 3. Schematic block diagram of the system (dc-link capacitor voltage difference dynamics) when the controller as well as the state observer are both implemented.

The balancing methods here presented are based on an asymptotic disturbance rejection approach. The proposed controllers estimate asymptotically the sinusoidal disturbances, having in common the idea of applying a control law to cancel them while regulating the capacitor voltage balance as well. The methods use some control concepts widely discussed in [25, 26,47-49], and also proposed for the neutral-point-clamped rectifier in [41,42], extending them to the multiple frequency approach of the back-to-back topology of the current paper.

In order to apply the control action, whereas vectors $\delta_{\alpha \beta, r}$ and $\delta_{\alpha \beta, i}$ are used to control the instantaneous powers, the remaining control inputs $\delta_{\gamma, r}$ and $\delta_{\gamma, i}$ are still free parameters, so they both can be used for the balancing of the capacitor voltages. However, in this section, a control signal denoted by $u_{\gamma}$ is defined containing both $\delta_{\gamma, r}$ and $\delta_{\gamma, i}$ as follows

$$
u_{\gamma}=k_{r} \delta_{\gamma, r}-k_{i} \delta_{\gamma, i} .
$$

As a result, the final equation of the voltage difference dynamics given by (12) is expressed as

$$
C \frac{d v_{d}}{d t}=u_{\gamma}+\phi_{r}+\phi_{i}
$$

The control term $u_{\gamma}$ is only introduced in the controller design stage to simplify the notation. The implementation of the definitive control law is discussed in the following section. Notice that (18) takes the form of the system considered in [27] for the linear setting, defining the disturbance $w$ in that reference as the sum of $\phi_{r}$ and $\phi_{i}$.

Fig. 3 depicts a schematic block diagram of (18) when the controller is included in the system. The block denoted by $P(s)$ is the transfer function defined in the Laplace domain by

$$
P(s)=\frac{1}{C s} .
$$

\subsection{State space estimation provided by a Luenberger observer}

The first proposed control method includes the asymptotic estimations of (15) and (16) provided by a state space observer, concretely by a Luenberger observer. Taking into account that the frequencies of the sinusoidal disturbances $\phi_{r}$ and $\phi_{i}$ are $3 \omega_{r}$ and $3 \omega_{i}$, respectively, (18) is expressed as the augmented system $\mathcal{S}$ described by

$$
\mathcal{S}:\left\{\begin{aligned}
\dot{x}_{d} & =\frac{1}{C} x_{\phi_{r}^{1}}+\frac{1}{C} x_{\phi_{i}^{1}}+\frac{1}{C} u_{\gamma} \\
\dot{x}_{\phi_{r}^{1}} & =x_{\phi_{r}^{2}} \\
\dot{x}_{\phi_{r}^{2}} & =-\left(3 \omega_{r}\right)^{2} x_{\phi_{r}^{1}} \\
\dot{x}_{\phi_{i}^{1}} & =x_{\phi_{i}^{2}} \\
\dot{x}_{\phi_{i}^{2}} & =-\left(3 \omega_{i}\right)^{2} x_{\phi_{i}^{1}} .
\end{aligned}\right.
$$


The capacitor voltage difference is defined by the variable $x_{d}$. The sinusoidal disturbances $\phi_{r}$ and $\phi_{i}$ by $x_{\phi_{r}^{1}}$ and $x_{\phi_{i}^{1}}$, and their time derivatives by $x_{\phi_{r}^{2}}$ and $x_{\phi_{i}^{2}}$, respectively. The control input of the system is $u_{\gamma}$.

Considering that the output of the system is the variable $x_{d}$, the state space representation of system $\mathcal{S}$ is given by

$$
\mathcal{S}:\left\{\begin{aligned}
\dot{\boldsymbol{x}} & =\boldsymbol{A} \boldsymbol{x}+\boldsymbol{B} u_{\gamma} \\
y & =\boldsymbol{C} \boldsymbol{x}
\end{aligned}\right.
$$

where $x \in \mathbb{R}^{5}$ is the state vector of the system, and it is defined by

$$
\boldsymbol{x}=\left[\begin{array}{lllll}
x_{d} & x_{\phi_{r}^{1}} & x_{\phi_{r}^{2}} & x_{\phi_{i}^{1}} & x_{\phi_{i}^{2}}
\end{array}\right]^{T},
$$

$y \in \mathbb{R}$ is the output, and $u_{\gamma} \in \mathbb{R}$ is the control input. The state matrix $\boldsymbol{A}$, and the input and output matrices, $\boldsymbol{B}$ and $\boldsymbol{C}$, respectively, are given by

$$
\boldsymbol{A}=\left[\begin{array}{ccccc}
0 & \frac{1}{C} & 0 & \frac{1}{C} & 0 \\
0 & 0 & 1 & 0 & 0 \\
0 & -\left(3 \omega_{r}\right)^{2} & 0 & 0 & 0 \\
0 & 0 & 0 & 0 & 1 \\
0 & 0 & 0 & -\left(3 \omega_{i}\right)^{2} & 0
\end{array}\right], \quad \boldsymbol{B}=\left[\begin{array}{c}
\frac{1}{C} \\
0 \\
0 \\
0 \\
0
\end{array}\right], \quad \boldsymbol{C}=\left[\begin{array}{c}
1 \\
0 \\
0 \\
0 \\
0
\end{array}\right] .
$$

Since the observability matrix of system $\mathcal{S}$ is full rank, the system is observable, and it is possible to reconstruct the system state from its output measurements via a state space observer. Thereby, to provide an estimate of $\boldsymbol{x}$, the Luenberger observer $\mathcal{S}_{O}$, assuming that the dc-link capacitor voltage difference is measurable, is designed as follows

$$
\mathcal{S}_{o}:\left\{\begin{array}{l}
\dot{\hat{\boldsymbol{x}}}=\boldsymbol{A} \hat{\boldsymbol{x}}+\boldsymbol{B} u_{\gamma}+\boldsymbol{L}(y-\hat{y}) \\
\hat{y}=\boldsymbol{C} \hat{\boldsymbol{x}} .
\end{array}\right.
$$

The estimated state vector $\dot{\hat{\boldsymbol{x}}} \in \mathbb{R}^{5}$ is given by

$$
\hat{\boldsymbol{x}}=\left[\begin{array}{lllll}
\hat{x}_{d} & \hat{x}_{\phi_{r}^{1}} & \hat{x}_{\phi_{r}^{2}} & \hat{x}_{\phi_{i}^{1}} & \hat{x}_{\phi_{i}^{2}}
\end{array}\right]^{T},
$$

the estimated output by $\hat{y} \in \mathbb{R}$, and the observer gain matrix is described by

$$
\boldsymbol{L}=\left[\begin{array}{lllll}
p_{1} & p_{2} & p_{3} & p_{4} & p_{5}
\end{array}\right]^{T} .
$$

The rest of the variables and parameters of (24) have been already defined in (21). The values of the elements of $\boldsymbol{L}$ are chosen in such a way that the poles of the observer $\mathcal{S}_{o}$ converge faster than the poles of system $\mathcal{S}$.

Finally, once the observer is implemented, the controller applies the estimates of $\phi_{r}$ and $\phi_{i}$ that the observer provides, represented by the state variables $\hat{x}_{\phi_{r}^{1}}$ and $\hat{x}_{\phi_{i}^{1}}$, respectively. Thus, the control law is defined by

$$
u_{\gamma}=k\left(v_{d}^{*}-v_{d}\right)-\hat{x}_{\phi_{r}^{1}}-\hat{x}_{\phi_{i}^{1}},
$$

where $k$ is a design constant parameter, and $v_{d}^{*}$ is the capacitor voltage difference reference, which is set to zero. Notice that the estimates $\hat{x}_{\phi_{r}^{1}}$ and $\hat{x}_{\phi_{i}^{1}}$ are applied to asymptotically cancel the terms $\phi_{r}$ and $\phi_{i}$ in (18), and they are implemented in parallel with a proportional controller to ensure that $v_{d}$ tends exponentially towards its reference. 


\subsection{Internal model principle based disturbance estimation}

The second approach is based on the internal model principle of control theory $[25,26]$, which states that the regulation of a system is achieved when the control law encapsulates, either implicity or explicitly, some representation of the process to be controlled. In this way, the feedback path should incorporate suitably reduplicated models of the dynamic structure of the disturbances.

Focusing on the capacitor voltage difference dynamics, some transfer functions are used to approximate the behavior of the sinusoidal disturbances. In this manner, the models of $\phi_{r}$ and $\phi_{i}$ consist of $\hat{\phi}_{r}$ and $\hat{\phi}_{i}$, respectively, which are defined in the Laplace domain as

$$
\begin{aligned}
& \hat{\phi}_{r}(s)=-g_{r} \frac{s}{s^{2}+\left(3 \omega_{r}\right)^{2}}\left(v_{d}^{*}(s)-v_{d}(s)\right) \\
& \hat{\phi}_{i}(s)=-g_{i} \frac{s}{s^{2}+\left(3 \omega_{i}\right)^{2}}\left(v_{d}^{*}(s)-v_{d}(s)\right),
\end{aligned}
$$

where $g_{r}$ and $g_{i}$ are positive constants, and $v_{d}^{*}$ is the capacitor voltage difference reference. Notice that the models of both disturbances have a pair of imaginary poles at the same frequency of the disturbances $\phi_{r}$ and $\phi_{i}$.

The disturbance approximations are applied to achieve the asymptotical cancellation of the disturbances and are constructed in parallel with a proportional controller in a feedback control system. Therefore, it leads to the following control law

$$
u_{\gamma}=k\left(v_{d}^{*}-v_{d}\right)-\hat{\phi}_{r}-\hat{\phi}_{i}
$$

where $k$ is the gain of the proportional controller.

\subsection{Disturbance estimation applying an adaptive algorithm}

Under the third control approach, an adaptive algorithm is introduced to asymptotically estimate the disturbances, in order to apply an adaptive feedforward cancellation at the input of the system of both $\phi_{r}$ and $\phi_{i}$, considering that their expressions are known.

The control method starts by proposing equivalent expressions for $\phi_{r}$ and $\phi_{i}$ applying, among others, the trigonometric identity

$$
\sqrt{a^{2}+b^{2}} \sin \left(\alpha+\arctan \left(\frac{b}{a}\right)\right)=a \sin (\alpha)+b \cos (\alpha) .
$$

Thereby, (15) and (16) are expressed as

$$
\begin{aligned}
\phi_{r} & =\eta_{1, r} \sin \left(3 \omega_{r} t\right)+\eta_{2, r} \cos \left(3 \omega_{r} t\right) \\
\phi_{i} & =\eta_{1, i} \sin \left(3 \omega_{i} t\right)+\eta_{2, i} \cos \left(3 \omega_{i} t\right) .
\end{aligned}
$$

The constants $\eta_{1, r}, \eta_{1, i}, \eta_{2, r}$ and $\eta_{2, i}$ are defined as follows

$$
\begin{aligned}
\eta_{1, r} & =\cos \left(3 \theta_{r}\right) \frac{\mu_{1, r}}{\sqrt{1+\mu_{2, r}^{2}}}-\sin \left(3 \theta_{r}\right) \frac{\mu_{1, r} \mu_{2, r}}{\sqrt{1+\mu_{2, r}^{2}}} \\
\eta_{2, r} & =\cos \left(3 \theta_{r}\right) \frac{\mu_{1, r} \mu_{2, r}}{\sqrt{1+\mu_{2, r}^{2}}}+\sin \left(3 \theta_{r}\right) \frac{\mu_{1, r}}{\sqrt{1+\mu_{2, r}^{2}}} \\
\eta_{1, i} & =\cos \left(3 \theta_{i}\right) \frac{\mu_{1, i}}{\sqrt{1+\mu_{2, i}^{2}}}-\sin \left(3 \theta_{i}\right) \frac{\mu_{1, i} \mu_{2, i}}{\sqrt{1+\mu_{2, i}^{2}}} \\
\eta_{2, i} & =\cos \left(3 \theta_{i}\right) \frac{\mu_{1, i} \mu_{2, i}}{\sqrt{1+\mu_{2, i}^{2}}}+\sin \left(3 \theta_{i}\right) \frac{\mu_{1, i}}{\sqrt{1+\mu_{2, i}^{2}}}
\end{aligned}
$$


The reason of introducing these new terms is that, considering sinusoidal functions, it is easier to estimate their amplitudes than their phases. Hence, with the goal of estimate both (32) and (33), their estimations denoted by $\hat{\phi}_{r}$ and $\hat{\phi}_{i}$, respectively, are defined in this control scheme as

$$
\begin{aligned}
& \hat{\phi}_{r}=\bar{\eta}_{1, r} \sin \left(3 \omega_{r} t\right)+\bar{\eta}_{2, r} \cos \left(3 \omega_{r} t\right) \\
& \hat{\phi}_{i}=\bar{\eta}_{1, i} \sin \left(3 \omega_{i} t\right)+\bar{\eta}_{2, i} \cos \left(3 \omega_{i} t\right) .
\end{aligned}
$$

The values of $\bar{\eta}_{1, r}, \bar{\eta}_{2, r}, \bar{\eta}_{1, i}$ and $\bar{\eta}_{2, i}$ are obtained proposing the following update laws

$$
\begin{aligned}
& \dot{\bar{\eta}}_{1, r}=-g_{r}\left(v_{d}^{*}-v_{d}\right) \sin \left(3 \omega_{r} t\right) \\
& \dot{\bar{\eta}}_{2, r}=-g_{r}\left(v_{d}^{*}-v_{d}\right) \cos \left(3 \omega_{r} t\right) \\
& \dot{\bar{\eta}}_{1, i}=-g_{i}\left(v_{d}^{*}-v_{d}\right) \sin \left(3 \omega_{i} t\right) \\
& \dot{\bar{\eta}}_{2, i}=-g_{i}\left(v_{d}^{*}-v_{d}\right) \cos \left(3 \omega_{i} t\right),
\end{aligned}
$$

where the adaptation gains are the constants $g_{r}$ and $g_{i}$.

As mentioned before, the estimations (38) and (39) are used to asymptotically cancel the disturbances. In this way, the control law here considered is exactly the same that the one defined for the previous control method. It is given by (30), where $\hat{\phi}_{r}$ and $\hat{\phi}_{i}$ are replaced by their estimations provided by the adaptive algorithm. Note that $k$ is the gain of the proportional controller implemented in parallel.

\section{Remark 1}

The definition of the proposed parameter update laws is based on the Lyapunov approach carried out in $[47,48]$, adopting it to the formulation of the final expression of the voltage difference dynamics given by (18). Thus, the first step of the approach considered is the definition of the vectors

$$
\begin{aligned}
\boldsymbol{\omega} & =\left[\begin{array}{llll}
\sin \left(3 \omega_{r} t\right) & \cos \left(3 \omega_{r} t\right) & \sin \left(3 \omega_{i} t\right) & \cos \left(3 \omega_{i} t\right)
\end{array}\right]^{T} \\
\widetilde{\boldsymbol{\eta}} & =\left[\begin{array}{llll}
\bar{\eta}_{1, r}-\eta_{1, r} & \bar{\eta}_{2, r}-\eta_{2, r} & \bar{\eta}_{1, i}-\eta_{1, i} & \bar{\eta}_{2, i}-\eta_{2, i}
\end{array}\right]^{T},
\end{aligned}
$$

to simplify in what follows the notation. Consequently, (18) is expressed, taking into account (32) and (33), and introducing the proposed control law in this section, as

$$
\dot{v}_{d}=\frac{1}{C}\left(k\left(v_{d}^{*}-v_{d}\right)-\widetilde{\boldsymbol{\eta}}^{\boldsymbol{T}} \boldsymbol{\omega}\right) .
$$

Then, the Lyapunov function candidate

$$
V=\frac{1}{2} C\left(v_{d}^{*}-v_{d}\right)^{2}+\frac{1}{2} \widetilde{\boldsymbol{\eta}}^{\boldsymbol{T}} \boldsymbol{\Gamma}^{-\mathbf{1}} \widetilde{\boldsymbol{\eta}}
$$

is proposed, where $\Gamma$ is the adaption gain matrix, which is a freely chosen symmetric positive definite matrix. The derivative over the time of $V$ is given by

$$
\dot{V}=-k\left(v_{d}^{*}-v_{d}\right)^{2}+\widetilde{\boldsymbol{\eta}}^{\boldsymbol{T}}\left(\boldsymbol{\Gamma}^{-1} \dot{\tilde{\boldsymbol{\eta}}}+\left(v_{d}^{*}-v_{d}\right) \boldsymbol{\omega}\right) .
$$

Choosing the vector

$$
\dot{\tilde{\boldsymbol{\eta}}}=-\boldsymbol{\Gamma}\left(v_{d}^{*}-v_{d}\right) \boldsymbol{\omega},
$$

it results in the expression

$$
\dot{V}=-k\left(v_{d}^{*}-v_{d}\right)^{2} \leq 0 .
$$


Applying LaSalle's invariance principle, it can be proven that the trajectories of the system tend to the point $\left(v_{d}, \widetilde{\boldsymbol{\eta}}\right)=\left(v_{d}^{*}, \mathbf{0}\right)$. Finally, the adaption gain matrix $\boldsymbol{\Gamma}$ is defined as follows

$$
\boldsymbol{\Gamma}=\left[\begin{array}{cccc}
g_{r} & 0 & 0 & 0 \\
0 & g_{r} & 0 & 0 \\
0 & 0 & g_{i} & 0 \\
0 & 0 & 0 & g_{i}
\end{array}\right] .
$$

Hence, considering (49), since the elements $\eta_{1, r}, \eta_{1, i}, \eta_{2, r}$ and $\eta_{2, i}$ of (45) are constant parameters, it leads to the update laws (40)-(43).

\subsection{Estimation of unknown frequency disturbances applying an adaptive algorithm}

In this fourth and last control method, the frequencies $3 \omega_{r}$ and $3 \omega_{i}$ of the sinusoidal disturbances (15) and (16) are assumed to be unknown constant values. Therefore, two new fixed parameters that should be estimated appear in the system. Thus, the estimations of the disturbances $\phi_{r}$ and $\phi_{i}$ are, respectively, defined by

$$
\begin{aligned}
\hat{\phi}_{r} & =\rho_{1, r} \cos \left(\varphi_{r}\right) \\
\hat{\phi}_{i} & =\rho_{1, i} \cos \left(\varphi_{i}\right) \\
\dot{\varphi}_{r} & =\rho_{2, r} \\
\dot{\varphi}_{i} & =\rho_{2, i},
\end{aligned}
$$

where $\rho_{1, r}$ and $\rho_{1, i}$ are the estimations of the magnitude of the disturbances, $\varphi_{r}$ and $\varphi_{i}$ the estimations of their phases, and $\rho_{2, r}$ and $\rho_{2, i}$ the estimations of their frequencies.

To obtain the values of the estimations, the approach presented in Section 4.3 is extended here by integrating a modified version of a phase-locked loop within the control scheme [49]. Thereby, the following update laws in the Laplace domain are considered

$$
\begin{aligned}
\rho_{1, r}(s) & =-\frac{1}{s} g_{1, r} y_{1, r}(s) \\
\rho_{2, r}(s) & =-\frac{1}{s} g_{2, r} \frac{s+a_{r}}{s+b_{r}} y_{2, r}(s) \\
\rho_{1, i}(s) & =-\frac{1}{s} g_{1, i} y_{1, i}(s) \\
\rho_{2, i}(s) & =-\frac{1}{s} g_{2, i} \frac{s+a_{i}}{s+b_{i}} y_{2, i}(s) .
\end{aligned}
$$

The parameters $g_{1, r}, g_{2, r}, g_{1, i}$ and $g_{2, i}$ are positive constants, while $a_{r}, b_{r}, a_{i}$ and $b_{i}$ are also constants chosen to guarantee the closed-loop stability of the system. Functions $y_{1, r}, y_{2, r}, y_{1, i}$ and $y_{2, i}$ are defined in the time domain as follows

$$
\begin{aligned}
& {\left[\begin{array}{l}
y_{1, r} \\
y_{2, r}
\end{array}\right]=\left[\begin{array}{cc}
P_{R}^{3 \omega_{r}} & -P_{I}^{3 \omega_{r}} \\
P_{I}^{3 \omega_{r}} & P_{R}^{3 \omega_{r}}
\end{array}\right]^{-1}\left[\begin{array}{r}
\left(v_{d}^{*}-v_{d}\right) \cos \left(\varphi_{r}\right) \\
-\left(v_{d}^{*}-v_{d}\right) \sin \left(\varphi_{r}\right)
\end{array}\right]} \\
& {\left[\begin{array}{l}
y_{1, i} \\
y_{2, i}
\end{array}\right]=\left[\begin{array}{cc}
P_{R}^{3 \omega_{i}} & -P_{I}^{3 \omega_{i}} \\
P_{I}^{3 \omega_{i}} & P_{R}^{3 \omega_{i}}
\end{array}\right]^{-1}\left[\begin{array}{r}
\left(v_{d}^{*}-v_{d}\right) \cos \left(\varphi_{i}\right) \\
-\left(v_{d}^{*}-v_{d}\right) \sin \left(\varphi_{i}\right)
\end{array}\right] .}
\end{aligned}
$$

The values of $P_{R}^{3 \omega_{r}}$ and $P_{I}^{3 \omega_{r}}$ are, respectively, the estimated real and imaginary parts of the frequency response of the system at the frequency of the disturbance $\phi_{r}$. For the disturbance $\phi_{i}$, 
they are denoted by $P_{R}^{3 \omega_{i}}$ and $P_{I}^{3 \omega_{i}}$. Accordingly, considering the transfer function (19), these parameters are given by

$$
\begin{aligned}
& P_{R}^{3 \omega_{r}}=\operatorname{Re}\left\{P\left(3 \omega_{r} j\right)\right\} \\
& P_{I}^{3 \omega_{r}}=\operatorname{Im}\left\{P\left(3 \omega_{r} j\right)\right\} \\
& P_{R}^{3 \omega_{i}}=\operatorname{Re}\left\{P\left(3 \omega_{i} j\right)\right\} \\
& P_{R}^{3 \omega_{i}}=\operatorname{Im}\left\{P\left(3 \omega_{i} j\right)\right\} .
\end{aligned}
$$

Note that the real parts of (19) at both disturbance frequencies are zero.

Finally, considering the estimations (52) and (53), they are implemented together with a proportional controller, whose positive gain is denoted by $k$, so the control law is described again by (30). It is also important to note that in this method, although the disturbance frequencies are unknown, the estimations of the response of the system (62)-(65) need an approximation of both frequencies. For this reason, the uncertainty of the frequencies have to be small enough to accurate a good estimation of the parameters.

\section{Remark 2}

The theoretical foundations of the adopted control method of this section were presented by Bodson et al., and they can be consulted in [49]. The approach is based on the steady-state response of a system to different sinusoidal inputs. Defining by $P_{s}(s)$ the plant transfer function of a timeinvariant linear system, and by $P_{s}(s)[x(t)]$ the time-domain output of the system when the input is the function of time $x(t)$, the following expression is satisfied in steady state

$$
P_{s}(s)\left[\begin{array}{c}
\cos \left(\omega_{o} t+\theta_{o}\right) \\
\sin \left(\omega_{o} t+\theta_{o}\right)
\end{array}\right]=\left[\begin{array}{rr}
\operatorname{Re}\left\{P_{s}\left(\omega_{o} j\right)\right\} & -\operatorname{Im}\left\{P_{s}\left(\omega_{o} j\right)\right\} \\
\operatorname{Im}\left\{P_{s}\left(\omega_{o} j\right)\right\} & \operatorname{Re}\left\{P_{s}\left(\omega_{o} j\right)\right\}
\end{array}\right]\left[\begin{array}{c}
\cos \left(\omega_{o} t+\theta_{o}\right) \\
\sin \left(\omega_{o} t+\theta_{o}\right)
\end{array}\right] .
$$

In the particular problem considered in this paper, the expression (18) derived from the mathematical analysis of Section 3 allows the definition of (19). This is the transfer function used to approximate the response of the system in steady state.

\section{Remark 3}

It is worth mentioning that the design of the controllers has been presented assuming that the values of the disturbance frequencies present a relevant difference between them. However, some problems may arise in some of the proposed control methods when the frequencies get very close to each other. For instance, the convergence of the estimations may be too slow, or the two frequency estimates could converge to a same value. Some methods to deal with this undesired situation are presented in $[36,50]$. Thus, in [36] the two sinusoidal disturbances are represented as a single sinusoid with time-varying parameters, while in [50] a frequency separation block is proposed to avoid the risk of convergence of the frequency estimates to the same value.

In addition, when the values of the disturbance frequencies are exactly the same, that is,

$$
3 \omega_{r}=3 \omega_{i}
$$

the sum of the disturbances $\phi_{r}$ and $\phi_{i}$ of (12) results in this case in an only pure sinusoidal disturbance whose frequency corresponds also with $3 \omega_{r}$ (or $3 \omega_{i}$, both are equivalent). This fact reduces the complexity of the proposed controller design since solely a disturbance should be asymptotically estimated and then rejected. In this way, the control methods discussed in [41,42], including some adjustments, can be used for the balancing of the dc-link capacitor voltages. For example, considering the internal model principle based method, only one transfer function should be applied to approximate the disturbance. 
Table II. Simulation parameters.

\begin{tabular}{ll}
\hline Parameter & Value \\
\hline Sampling time $\left(T_{s}\right)$ & $100 \mu \mathrm{s}$ \\
Rectifier instantaneous reactive power reference $\left(q_{r}^{*}\right)$ & $0 \mathrm{VAr}$ \\
Inverter instantaneous active power reference $\left(p_{i}^{*}\right)$ & $10 \mathrm{~kW}$ \\
Inverter instantaneous reactive power reference $\left(q_{i}^{*}\right)$ & $0 \mathrm{VAr}$ \\
Rectifier phase voltage frequency $\left(\omega_{r}\right)$ & $2 \pi \cdot 50 \mathrm{rad} / \mathrm{s}$ \\
Inverter phase voltage frequency $\left(\omega_{i}\right)$ & $2 \pi \cdot 60 \mathrm{rad} / \mathrm{s}$ \\
Rectifier phase voltage amplitude $\left(\left|\boldsymbol{v}_{\boldsymbol{\alpha} \boldsymbol{\beta}, \boldsymbol{r}}\right|\right)$ & $380 \mathrm{~V}$ \\
Inverter phase voltage amplitude $\left(\left|\boldsymbol{v}_{\boldsymbol{\alpha} \boldsymbol{\beta}, \boldsymbol{i}}\right|\right)$ & $380 \mathrm{~V}$ \\
Inductors $\left(L_{r}, L_{i}\right)$ & $5 \mathrm{mH}$ \\
Capacitors $\left(C_{1}, C_{2}\right)$ & $1100 \mu \mathrm{F}$ \\
\hline
\end{tabular}

\section{IMPLEMENTATION OF THE CONTROL LAW}

As pointed out in the preceding section, once the control law $u_{\gamma}$ is calculated applying any one of the proposed controllers, it should be determined the way (17) is implemented. For this purpose, control inputs $\delta_{\gamma, r}$ and $\delta_{\gamma, i}$ are available to be used for the balancing of the dc-link capacitor voltages.

Since in the back-to-back topology both rectifier and inverter converter stages are usually decoupled for control purposes, i.e., they are controlled individually, the first possible implementation leads to set one of the control inputs to zero while devoting the remaining one to the voltage balance control. Thereby, it yields the expressions

$$
\begin{gathered}
\delta_{\gamma, i}=0 \rightarrow \delta_{\gamma, r}=\frac{u_{\gamma}}{k_{r}} \\
\delta_{\gamma, r}=0 \rightarrow \delta_{\gamma, i}=-\frac{u_{\gamma}}{k_{i}} .
\end{gathered}
$$

With this approach, only one of the converter stages deals with the capacitor voltage balancing, so either the rectifier or the inverter should apply the control algorithm.

Then, the control action implemented considering (68) or (69) is modulated together with rectifier and inverter control terms $\boldsymbol{\delta}_{\boldsymbol{\alpha} \beta, r}$ and $\boldsymbol{\delta}_{\boldsymbol{\alpha} \boldsymbol{\beta}, i}$, after they all are transformed into abc coordinates, to provide the feasible gating signals. However, it could be possible that, in case that the values of $\delta_{\gamma, r}$ or $\delta_{\gamma, r}$ were not close enough to zero, the gating signals may not be correctly generated. In view of this, to cope with this saturation problem, the second approach proposes the use of both control inputs $\delta_{\gamma, r}$ and $\delta_{\gamma, i}$ to implement the control law $u_{\gamma}$, producing the same effect as applying only one of them. Thus, the second approach is described as follows

$$
\begin{aligned}
& \delta_{\gamma, r}=\frac{u_{\gamma}}{2 k_{r}} \\
& \delta_{\gamma, i}=-\frac{u_{\gamma}}{2 k_{i}},
\end{aligned}
$$

where both $\delta_{\gamma, r}$ and $\delta_{\gamma, i}$ are used to provide the previously calculated control law. Because $k_{r}$ and $k_{i}$ do not present always the same value, it is not a distribution in equal shares but it is the easier one to implement. Furthermore, it requires less computational time than other possible strategies. Therefore, even though under this approach there is no complete control decoupling of the inverter and rectifier, this method is the one considered in the simulations carried out and presented next. 
Table III. Design constants of the instantaneous power controllers and of the total dc-link voltage controller.

\begin{tabular}{ll}
\hline Design constant & Value \\
\hline Rectifier instantaneous power controller constants $\left(k_{p, r}, k_{q, r}\right)$ & $4 \cdot 10^{-7}$ \\
Inverter instantaneous power controller constants $\left(k_{p, i}, k_{q, i}\right)$ & $4 \cdot 10^{-7}$ \\
Total dc-link voltage proportional gain $\left(k_{p r}\right)$ & 0.005 \\
Total dc-link voltage integral gain $\left(k_{i n t}\right)$ & 0.05 \\
\hline
\end{tabular}

Table IV. Design constants of the proposed voltage balancing methods.

\begin{tabular}{|c|c|c|}
\hline Controller & Constant & Value \\
\hline Observer-based estimation controller & $k$ & 10 \\
\hline \multirow{2}{*}{ Internal model principle based disturbance estimation controller } & $k$ & 10 \\
\hline & $g_{r}, g_{i}$ & 1000 \\
\hline \multirow{2}{*}{ Controller with disturbance estimation applying an adaptive algorithm } & $\int k$ & 10 \\
\hline & $g_{r}, g_{i}$ & 1000 \\
\hline \multirow{5}{*}{$\begin{array}{l}\text { Controller with estimation of unknown frequency disturbances } \\
\text { applying an adaptive algorithm }\end{array}$} & $(k$ & 10 \\
\hline & $g_{1, r}, g_{1, i}$ & 140 \\
\hline & $g_{2, r}, g_{2, i}$ & 200 \\
\hline & $a_{r}, a_{i}$ & 5 \\
\hline & $b_{r}, b_{i}$ & 30 \\
\hline
\end{tabular}

\section{SIMULATION RESULTS}

In this section, the simulations results, obtained with Simulink under Matlab environment, are shown and discussed. The equations that describe the model of the system [44] together with the voltage balancing methods proposed in Section 4 have been implemented to study the behavior of the system and evaluate its performance. In addition, the instantaneous power controller and the total dc-link voltage controller have been designed and implemented as well. Particularly, the simulations are focused on the transient stage of the capacitor voltage difference of the converter dc-link when any of the system parameters changes abruptly its value. Moreover, the aim of the simulations is also to validate the different voltage balance controllers.

For this purpose, some simulations have been carried out applying each one of the four proposed control methods previously mentioned, considering a total voltage reference step in the dc-link. To that end, the reference $v_{s}^{*}$ is moved from $800 \mathrm{~V}$ to $700 \mathrm{~V}$. The values of the parameters used in the simulations are summarized in Table II. The phase voltage frequencies $\omega_{r}$ and $\omega_{i}$ are assumed to remain constant during the simulations, which have been implemented in discrete time with sampling time set to $100 \mu \mathrm{s}$.

Focusing on the instantaneous powers, the control method proposed in [46] is adopted here, which is based on the output regulation subspaces and implements a proportional-type direct power control. Thereby, the control vectors $\delta_{\alpha \beta, r}$ and $\delta_{\alpha \beta, i}$ are applied, respectively, to control the instantaneous powers of the rectifier and of the inverter. Concerning the regulation of the total dclink voltage, the rectifier instantaneous active power reference is used to drive the voltage towards its reference. Considering (11), i.e., the expression of the total dc-link voltage dynamics under Assumption 1, a standard proportional-integral (PI) controller is designed as follows

$$
p_{r}^{*}=k_{p r}\left(v_{d c}^{* 2}-v_{d c}^{2}\right)+k_{i n t} \int_{0}^{t}\left(v_{d c}^{* 2}-v_{d c}^{2}\right) d \tau,
$$




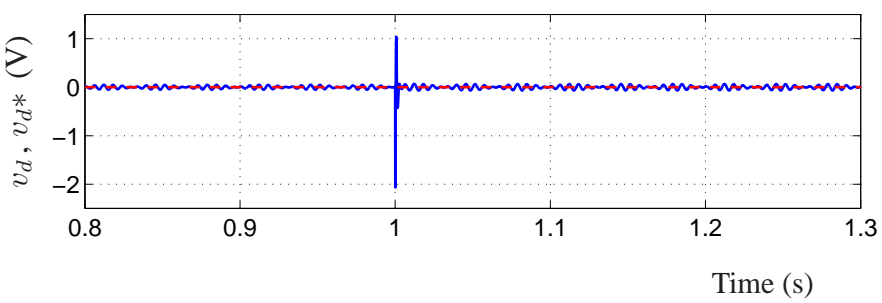

(a)

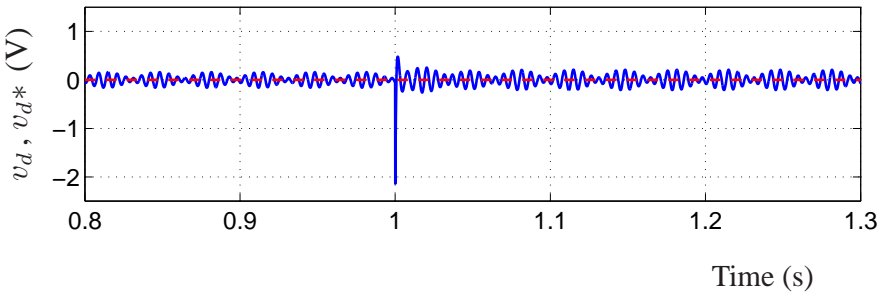

(b)

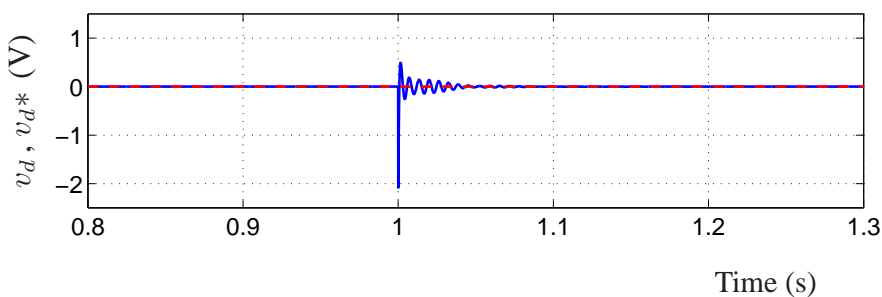

(c)

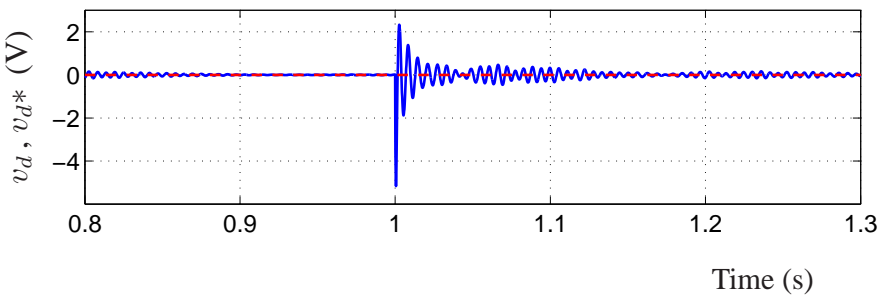

(d)

Figure 4. From top to bottom, difference between the dc-link capacitor voltages $v_{d}$ (solid), when a total voltage reference step occurs, and its reference value $v_{d}^{*}$ (dashed), applying the control methods: (a) Observer-based estimation controller. (b) Internal model principle based disturbance estimation controller. (c) Controller with disturbance estimation applying an adaptive algorithm. (d) Controller with disturbance estimation of unknown frequency disturbances applying an adaptive algorithm.

and it is introduced in the system. Constants $k_{p r}$ and $k_{i n t}$ are the tuning parameters of the controller.

Regarding the design constants of the controllers, their values have been chosen in such a way that the time-scale assumptions mentioned in Section 3 are well grounded. Table III illustrates the design constants of the instantaneous power controllers as well as those of the total dc-link voltage controller. The parameters of the voltage balance controllers are summarized in Table IV. These control methods have been presented previously in continuous time, so to work out the simulations, they have been discretized considering the sampling time shown in Table II.

Fig. 4 depicts the evolution of the variable $v_{d}$, that is, the evolution of the voltage difference between the dc-link capacitors, when the total dc-link voltage reference changes at $t=1 \mathrm{~s}$. The four control methods present a similar behavior. The voltage difference begins to oscillate, achieving its reference, with is set to zero, quickly. The controller that estimates the disturbances considering 


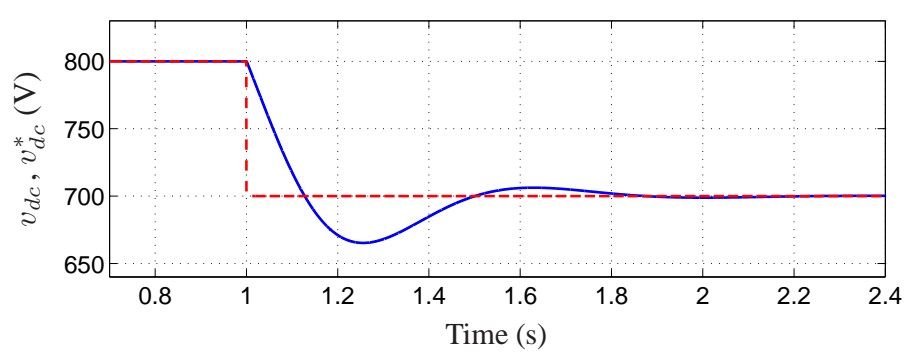

Figure 5. Evolution of the total dc-link voltage (solid) and its reference (dashed).

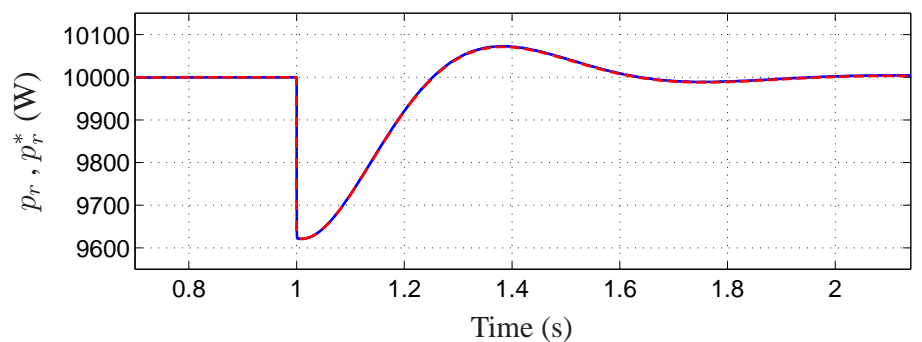

Figure 6. Behavior of the rectifier instantaneous active power $p_{r}$ (solid) and its reference $p_{r}^{*}$ (dashed).

that their frequencies are unknown shows the worst voltage balancing, since it contains a higher number of parameters to estimate (the frequencies are also estimated parameters applying this control method). However, the voltage difference still remains close to zero. Nevertheless, if the frequencies $\omega_{r}$ and $\omega_{r}$ change their nominal values (but not much because the terms $P_{R}^{3 \omega_{r}}, P_{I}^{3 \omega_{r}}$, $P_{R}^{3 \omega_{i}}$ and $P_{I}^{3 \omega_{i}}$ of (60) and (61) have been estimated and set considering the nominal values of the disturbance frequencies), this controller provides a better estimation of the disturbances than the one provided by the other voltage balance controllers, which have been designed for specific values of $\omega_{r}$ and $\omega_{i}$ (Table II). Focusing on the steady state behavior, the voltage difference presents a very small ripple, which is lower than $0.5 \mathrm{~V}$ and is not relevant. Hence, the variable $v_{d}$ is properly regulated to zero with all the proposed control methods, so a good voltage balancing is ensured.

Concerning the behavior of the total dc-link voltage in the simulation carried out implementing the controller of Section 4.1, note that, when the voltage reference step occurs, the variable $v_{d c}$ tends also correctly towards its new reference (Fig. 5). As mentioned before, this variable is regulated applying the instantaneous powers of the rectifier. Concretely, via the instantaneous active power reference $p_{r}^{*}$, whose evolution is illustrated in Fig. 6. The power variable $p_{r}$ is also depicted in the figure to illustrate the usefulness of the instantaneous power controller. Notice that its behavior is practically identical to the one of $p_{r}^{*}$, and it is difficult to distinguish $p_{r}$ from $p_{r}^{*}$.

Moving on to the control method that estimates unknown frequency sinusoidal disturbances (Section 4.4), Fig. 7 illustrates the evolution of the frequency estimations of the rectifier and inverter phase voltages. They are denoted by, respectively, the variables $\rho_{2, r}$ and $\rho_{2, i}$. The initial states of both variables have been set to $\rho_{2, r}(0)=941 \mathrm{rad} / \mathrm{s}$ and $\rho_{2, i}(0)=1130 \mathrm{rad} / \mathrm{s}$. As stated in Section 3.2 , the frequencies of the sinusoidal disturbances $\phi_{r}$ and $\phi_{i}$ are the triple frequencies of those of the rectifier and inverter phase voltages, respectively. Since the values of the phase voltage frequencies in the simulations are $\omega_{r}=2 \pi \cdot 50 \mathrm{rad} / \mathrm{s}$ and $\omega_{i}=2 \pi \cdot 60 \mathrm{rad} / \mathrm{s}$, notice that both $\rho_{2, r}$ and $\rho_{2, i}$ tend properly to their specific references $\left(\rho_{2, r}^{*}=3 \omega_{r} \simeq 942.48 \mathrm{rad} / \mathrm{s}\right.$ and $\rho_{2, i}^{*}=3 \omega_{i} \simeq 1130.97 \mathrm{rad} / \mathrm{s}$ ), even when the total voltage reference step occurs.

Finally, Fig. 8 shows the amplitude frequency spectrums of the dc-link capacitor voltage difference. On one hand, when the observer-based disturbance estimation controller is implemented to regulate the voltage balance. On the other hand, when a traditional PI controller is introduced in the system with the purpose of achieving the same control aim. Notice that both spectrums present the highest frequency content at the frequencies of the disturbances $\left(3 f_{r}=150 \mathrm{~Hz}, 3 f_{i}=180 \mathrm{~Hz}\right)$. 


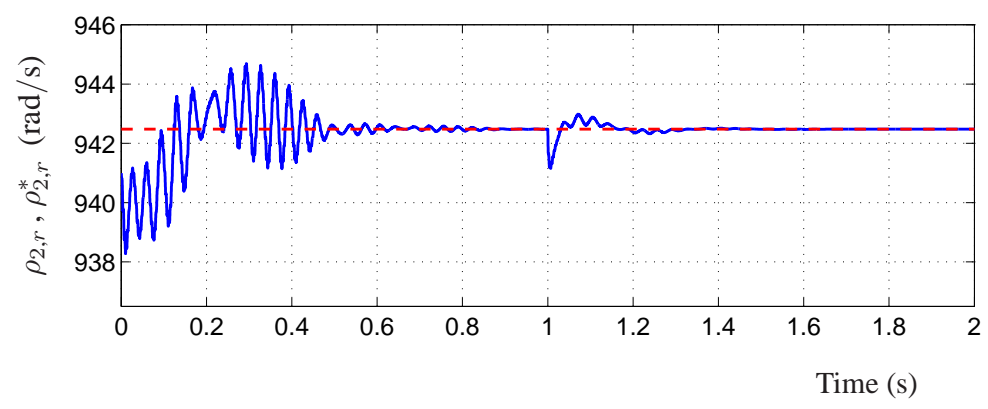

(a)

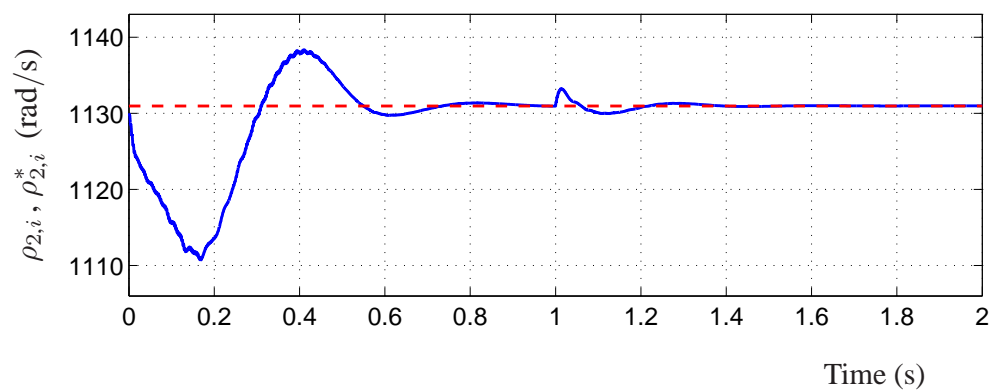

(b)

Figure 7. Frequency estimations provided by the controller with estimation of unknown frequency disturbances applying an adaptive algorithm. From top to bottom: (a) Rectifier frequency estimation variable $\rho_{2, r}$ (solid) and its reference value $\rho_{2, r}^{*}$ (dashed). (b) Inverter frequency estimation variable $\rho_{2, i}$ (solid) and its reference value $\rho_{2, i}^{*}$ (dashed).

Nevertheless, the magnitude obtained applying the first control method is much smaller than that provided considering the PI controller. Therefore, the proposed control methods in this work present the important benefit of a smaller frequency content of the dc-link capacitor voltage difference, in contrast with the use of other voltage balancing strategies (the spectrum features of $v_{d}$ considering the remaining control methods presented in Section 4 are analogous to the one obtained applying the observer).

\section{CONCLUSIONS}

In this work, several control methods designed to achieve the balancing of the dc-link capacitor voltages in three-phase three-level diode-clamped back-to-back power converters have been presented. Under some time-scale assumptions, the dynamics of the capacitor voltage difference are analyzed, resulting in a expression that contains several complex terms, which are approximated by sinusoidal functions of time. The significance of this fact is highly relevant, since it is the main idea behind the design of the proposed controllers, which are based on the estimations of these sinusoidal functions, considering them as external disturbances of the system. In this way, the paper relates the capacitor voltage imbalance problem to the problem of regulating the output of the system, under the presence of periodic disturbances that should be canceled.

The simulations presented in Section 6 have proved the usefulness of the voltage balance controllers. It is worth stressing that the control laws are not difficult to implement. Due to the disturbance frequencies, which are three times the frequencies of the rectifier and inverter side phase voltages, the voltage balancing methods have been designed and introduced in the system taking into account the values of the frequencies used in the simulations (see Table II). If other nominal values of the frequencies are considered, the controllers should be redesigned to cancel the disturbances, since the disturbance frequencies are modified and present other values. 


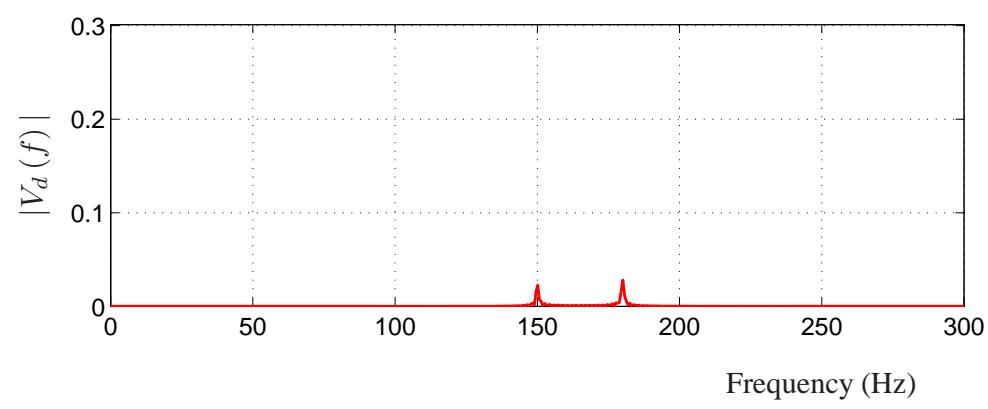

(a)

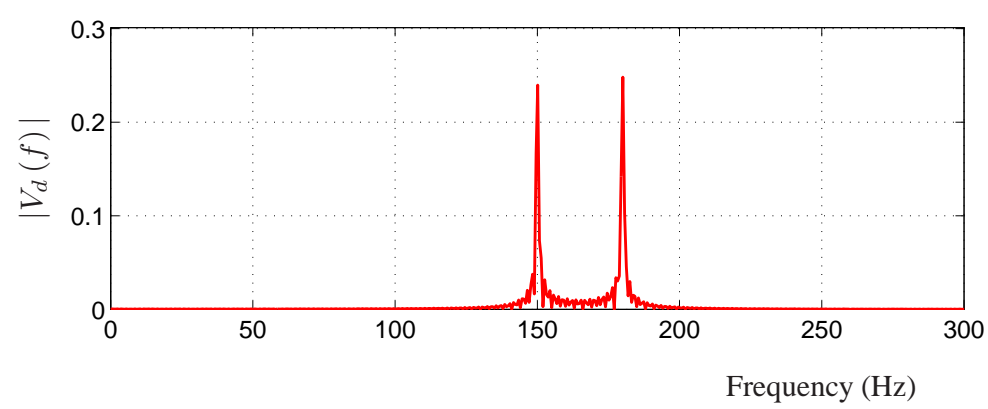

(b)

Figure 8. From top to bottom, single-sided amplitude frequency spectrums of the dc-link capacitor voltage difference $v_{d}$, applying the control methods: (a) Controller that estimates the disturbances via a Luenberger observer. (b) Traditional PI controller.

\section{A. APPENDIX}

\section{Mathematical analysis of the capacitor voltage difference dynamics of the converter}

Focusing on the capacitor voltage difference dynamics defined by (1), notice that they are expressed as a function of the components of control vectors $\delta_{\alpha \beta \gamma, r}$ and $\delta_{\alpha \beta \gamma, i}$, and of the phase current vectors $\boldsymbol{i}_{\boldsymbol{\alpha} \boldsymbol{\beta}, \boldsymbol{r}}$ and $\boldsymbol{i}_{\boldsymbol{\alpha} \boldsymbol{\beta}, \boldsymbol{i}}$. Since Assumption 1 is related to the power dynamics, it is more suitable for the analysis to express (1) in terms of power instead of current. Thereby, considering (7)-(10), the phase currents are approximated by

$$
\begin{aligned}
i_{\alpha, r} & \simeq \frac{p_{r}^{*}}{\left|\boldsymbol{v}_{\boldsymbol{\alpha} \boldsymbol{\beta}, \boldsymbol{r}}\right|^{2}} v_{\alpha, r}-\frac{q_{r}^{*}}{\left|\boldsymbol{v}_{\boldsymbol{\alpha} \boldsymbol{\beta}, \boldsymbol{r}}\right|^{2}} v_{\beta, r} \\
i_{\beta, r} & \simeq \frac{p_{r}^{*}}{\left|\boldsymbol{v}_{\boldsymbol{\alpha} \boldsymbol{\beta}, \boldsymbol{r}}\right|^{2}} v_{\beta, r}+\frac{q_{r}^{*}}{\left|\boldsymbol{v}_{\boldsymbol{\alpha} \boldsymbol{\beta}, \boldsymbol{r}}\right|^{2}} v_{\alpha, r} \\
i_{\alpha, i} & \simeq \frac{p_{i}^{*}}{\left|\boldsymbol{v}_{\boldsymbol{\alpha} \boldsymbol{\beta}, i}\right|^{2}} v_{\alpha, i}-\frac{q_{i}^{*}}{\left|\boldsymbol{v}_{\boldsymbol{\alpha} \boldsymbol{\beta}, i}\right|^{2}} v_{\beta, i} \\
i_{\beta, i} & \simeq \frac{p_{i}^{*}}{\left|\boldsymbol{v}_{\boldsymbol{\alpha} \boldsymbol{\beta}, i}\right|^{2}} v_{\beta, i}+\frac{q_{i}^{*}}{\left|\boldsymbol{v}_{\boldsymbol{\alpha} \boldsymbol{\beta}, \boldsymbol{i}}\right|^{2}} v_{\alpha, i}
\end{aligned}
$$

defining this well-known change of variables in three-phase circuits. It is important to mention that the phase voltages in $\alpha \beta$ coordinates are expressed as

$$
\begin{aligned}
& v_{\alpha, r}=\left|\boldsymbol{v}_{\boldsymbol{\alpha} \boldsymbol{\beta}, \boldsymbol{r}}\right| \cos \left(\omega_{r} t+\theta_{r}\right) \\
& v_{\beta, r}=\left|\boldsymbol{v}_{\boldsymbol{\alpha} \boldsymbol{\beta}, \boldsymbol{r}}\right| \sin \left(\omega_{r} t+\theta_{r}\right) \\
& v_{\alpha, i}=\left|\boldsymbol{v}_{\boldsymbol{\alpha} \boldsymbol{\beta}, \boldsymbol{i}}\right| \cos \left(\omega_{i} t+\theta_{i}\right)
\end{aligned}
$$




$$
v_{\beta, i}=\left|\boldsymbol{v}_{\boldsymbol{\alpha} \boldsymbol{\beta}, i}\right| \sin \left(\omega_{i} t+\theta_{i}\right),
$$

where $\omega_{r}$ and $\omega_{i}$ are the frequencies of the phase voltages in the rectifier and inverter sides of the system, respectively. Their phases are denoted by $\theta_{r}$ and $\theta_{i}$.

In the same way, concerning the control vectors $\delta_{\alpha \beta, r}$ and $\delta_{\alpha \beta, i}$ (devoted to regulate the instantaneous powers), their expressions are obtained introducing (73)-(76) in the phase current dynamics [44], assuming that the capacitor voltage imbalance is small, i.e., $v_{d} \simeq 0$. Consequently, when the instantaneous powers are located around their desired values, they are described by

$$
\begin{aligned}
& \boldsymbol{\delta}_{\boldsymbol{\alpha} \boldsymbol{\beta}, \boldsymbol{r}} \simeq \frac{2}{v_{d c}} \lambda_{1, r} \boldsymbol{v}_{\boldsymbol{\alpha} \boldsymbol{\beta}, \boldsymbol{r}}-\frac{2}{v_{d c}} \lambda_{2, r} \boldsymbol{J} \boldsymbol{v}_{\boldsymbol{\alpha} \boldsymbol{\beta}, \boldsymbol{r}} \\
& \boldsymbol{\delta}_{\boldsymbol{\alpha} \boldsymbol{\beta}, \boldsymbol{i}} \simeq \frac{2}{v_{d c}} \lambda_{1, i} \boldsymbol{v}_{\boldsymbol{\alpha} \boldsymbol{\beta}, \boldsymbol{i}}+\frac{2}{v_{d c}} \lambda_{2, i} \boldsymbol{J} \boldsymbol{v}_{\boldsymbol{\alpha} \boldsymbol{\beta}, \boldsymbol{i}} .
\end{aligned}
$$

Constant parameters $\lambda_{1, r}, \lambda_{2, r}, \lambda_{1, i}, \lambda_{2, i}$ as well as the matrix $\boldsymbol{J}$ are introduced to simplify the notation. They are defined as follows

$$
\begin{aligned}
\lambda_{1, r} & =1+\frac{L_{r} \omega_{r} q_{r}^{*}}{\left|\boldsymbol{v}_{\boldsymbol{\alpha} \boldsymbol{\beta}, \boldsymbol{r}}\right|^{2}} \\
\lambda_{2, r} & =\frac{L_{r} \omega_{r} p_{r}^{*}}{\left|\boldsymbol{v}_{\boldsymbol{\alpha} \boldsymbol{\beta}, \boldsymbol{r}}\right|^{2}} \\
\lambda_{1, i} & =1-\frac{L_{i} \omega_{i} q_{i}^{*}}{\left|\boldsymbol{v}_{\boldsymbol{\alpha} \boldsymbol{\beta}, \boldsymbol{i}}\right|^{2}} \\
\lambda_{2, i} & =\frac{L_{i} \omega_{i} p_{i}^{*}}{\left|\boldsymbol{v}_{\boldsymbol{\alpha} \boldsymbol{\beta}, \boldsymbol{i}}\right|^{2}} \\
\boldsymbol{J} & =\left[\begin{array}{rr}
0 & -1 \\
1 & 0
\end{array}\right] .
\end{aligned}
$$

Moving on to the dynamics under analysis, introducing the change of variables (73)-(76) as well as the control vectors (81) and (82) in (1), the variation over the time of the voltage difference results in the complex expression

$$
\begin{aligned}
C \frac{d v_{d}}{d t}= & \frac{2 p_{r}^{*}}{\sqrt{3} v_{d c}} \delta_{\gamma}^{r}-\frac{2 p_{i}^{*}}{\sqrt{3} v_{d c}} \delta_{\gamma}^{i} \\
& +\frac{2 p_{r}^{*} v_{\alpha, r}-2 q_{r}^{*} v_{\beta, r}}{\sqrt{6}\left|\boldsymbol{v}_{\boldsymbol{\alpha} \boldsymbol{\beta}, \boldsymbol{r}}\right|^{2} v_{d c}^{2}}\left(\left(\lambda_{1, r} v_{\alpha, r}+\lambda_{2, r} v_{\beta, r}\right)^{2}-\left(\lambda_{1, r} v_{\beta, r}-\lambda_{2, r} v_{\alpha, r}\right)^{2}\right) \\
& -\frac{4 p_{r}^{*} v_{\beta, r}+4 q_{r}^{*} v_{\alpha, r}}{\sqrt{6}\left|\boldsymbol{v}_{\boldsymbol{\alpha} \boldsymbol{\beta}, \boldsymbol{r}}\right|^{2} v_{d c}^{2}}\left(\lambda_{1, r} v_{\alpha, r}+\lambda_{2, r} v_{\beta, r}\right)\left(\lambda_{1, r} v_{\beta, r}-\lambda_{2, r} v_{\alpha, r}\right) \\
& +\frac{2 q_{i}^{*} v_{\beta, i}-2 p_{i}^{*} v_{\alpha, i}}{\sqrt{6}\left|\boldsymbol{v}_{\boldsymbol{\alpha} \boldsymbol{\beta}, i}\right|^{2} v_{d c}^{2}}\left(\left(\lambda_{1, i} v_{\alpha, i}-\lambda_{2, i} v_{\beta, i}\right)^{2}-\left(\lambda_{1, i} v_{\beta, i}+\lambda_{2, i} v_{\alpha, i}\right)^{2}\right) \\
& +\frac{4 p_{i}^{*} v_{\beta}^{i}+4 q_{i}^{*} v_{\alpha, i}}{\sqrt{6}\left|\boldsymbol{v}_{\boldsymbol{\alpha} \boldsymbol{\beta}, i}\right|^{2} v_{d c}^{2}}\left(\lambda_{1, i} v_{\alpha, i}-\lambda_{2, i} v_{\beta, i}\right)\left(\lambda_{1, i} v_{\beta, i}+\lambda_{2, i} v_{\alpha, i}\right) .
\end{aligned}
$$

Nonetheless, according to the phase voltage definitions (77)-(80), and considering some trigonometric identities and the constants $\mu_{1, r}, \mu_{2, r}, \mu_{1, i}$ and $\mu_{2, i}$ given by

$$
\mu_{1, r}=2 \frac{\left|\boldsymbol{v}_{\boldsymbol{\alpha} \boldsymbol{\beta}, \boldsymbol{r}}\right|}{\sqrt{6} v_{d c}^{2}}\left(\lambda_{1, r}^{2}+\lambda_{2, r}^{2}\right) \sqrt{p_{r}^{* 2}+q_{r}^{* 2}}
$$




$$
\begin{aligned}
& \mu_{2, r}=\frac{\left(\lambda_{1, r}^{2}-\lambda_{2, r}^{2}\right) p_{r}^{*}+2 \lambda_{1, r} \lambda_{2, r} q_{r}^{*}}{-\left(\lambda_{1, r}^{2}-\lambda_{2, r}^{2}\right) q_{r}^{*}+2 \lambda_{1, r} \lambda_{2, r} p_{r}^{*}} \\
& \mu_{1, i}=2 \frac{\left|\boldsymbol{v}_{\boldsymbol{\alpha} \boldsymbol{\beta}, i}\right|}{\sqrt{6} v_{d c}^{2}}\left(\lambda_{1, i}^{2}+\lambda_{2, i}^{2}\right) \sqrt{p_{i}^{* 2}+q_{i}^{* 2}} \\
& \mu_{2, i}=-\left(\lambda_{1, i}^{2}-\lambda_{2, i}^{2}\right) p_{i}^{*}+2 \lambda_{1, i} \lambda_{2, i} q_{i}^{*} \\
&\left(\lambda_{1, i}^{2}-\lambda_{2, i}^{2}\right) q_{i}^{*}+2 \lambda_{1, i} \lambda_{2, i} p_{i}^{*}
\end{aligned}
$$

from (88) the reduced expression defined by (12) is derived. Hence, (12) is the final expression of the analysis worked out.

\section{ACKNOWLEDGEMENTS}

This work has been funded under grants MICINN-FEDER DPI2009-09661 and Junta de Andalucía P07TIC-02991.

\section{REFERENCES}

1. Kouro S, Malinowski M, Gopakumar K, Pou J, Franquelo LG, Wu B, Rodríguez J, Pérez MA, León JI. Recent advances and industrial applications of multilevel converters. IEEE Transactions on Industrial Electronics 2010; 57(8):2553-2580

2. Rodríguez J, Pontt J, Alzamora G, Becker N, Einenkel O, Weinstein A. Novel 20-MW downhill donveyor system using three-level converters. IEEE Transactions on Industrial Electronics 2002; 49(5):1093-1100.

3. Kohlmeier H, Niermeyer O, Schröder D. Highly dynamic four-quadrant AC motor drive with improved power factor and on-line optimized pulse pattern with PROMC. IEEE Transactions on Industry Applications 1987; IA23(6):1001-1009.

4. Ishida T, Matsuse K, Miyamoto T, Sasagawa K, Huang L. Fundamental characteristics of five-level double converters with adjustable DC voltages for induction motor drives. IEEE Transactions on Industrial Electronics 2002; 49(4):775-782.

5. Alepuz S, Busquets-Monge S, Bordonau J, Martínez-Velasco JA, Silva CA, Pontt J, Rodríguez J. Control strategies based on symmetrical components for grid-connected converters under voltage dips. IEEE Transactions on Industrial Electronics 2009; 56(6):2162-2173.

6. Calle A, Rocabert J, Busquets-Monge S, Bordonau J, Alepuz S, Peracaula J. Three-level three-phase neutral-pointclamped back-to-back converter applied to a wind emulator. Proceedings of the 13th European Conference on Power Electronics and Applications EPE 2009, Barcelona, Spain, 2009.

7. Carrasco JM, Franquelo LG, Bialasiewicz JT, Galván E, Portillo R, Prats MM, León JI, Moreno-Alfonso N. Powerelectronic systems for the grid integration of renewable energy sources: a survey. IEEE Transactions on Industrial Electronics 2006; 53(4):1002-1016.

8. Liserre M, Cárdenas R, Molinas M, Rodríguez J. Overview of multi-MW wind turbines and wind parks. IEEE Transactions on Industrial Electronics 2011; 58(4):1081-1095.

9. Kolar JW, Friedli T, Rodríguez J, Wheeler PW. Review of three-phase PWM AC-AC converter topologies. IEEE Transactions on Industrial Electronics 2011; 58(11):4988-5006.

10. Kolar JW, Friedli T, Krismer F, Round SD, The essence of three-phase AC/AC converter systems. Proceedings of the 13th Power Electronics and Motion Control Conference EPE-PEMC 2008, Poznan, Poland, 2008; 27-42.

11. Friedli T, Kolar JW. Comprehensive comparison of three-phase AC-AC matrix converter and voltage DC-link backto-back converter systems. Proceedings of the International Power Electronics Conference IPEC 2010, Sapporo, Japan, 2010; 2789-2798.

12. Nabae A, Takahashi I, Akagi H. A new neutral-point clamped PWM inverter. IEEE Transactions on Industry Applications 1981; IA-17(5):518-523

13. Rodríguez J, Bernet S, Wu B, Pontt JO, Kouro S. Multilevel voltage-source-converter topologies for industrial medium-voltage drives. IEEE Transactions on Industrial Electronics 2007; 54(6):2930-2945.

14. Abu-Rub H, Holtz J, Rodríguez J, Baoming G. Medium-voltage multilevel converters-state of the art, challenges, and requirements in industrial applications. IEEE Transactions on Industrial Electronics 2010; 57(8):2581-2596.

15. Franquelo LG, Rodríguez J, León JI, Kouro S, Portillo R, Prats MM. The age of multilevel converters arrives. IEEE Industrial Electronics Magazine 2008; 2(2):28-39.

16. Marchesoni M, Tenca P. Theoretical and practical limits in multilevel MPC inverters with passive front ends. Proceedings of the 9th European Conference on Power Electronics and Applications EPE 2001, Graz, Austria, 2001.

17. Pou J, Pindado R, Boroyevich D. Voltage-balance limits in four-level diode-clamped converters with passive front ends. IEEE Transactions on Industrial Electronics 2005; 52(1):190-196.

18. Marchesoni M, Tenca P. Diode-clamped multilevel converters: a practicable way to balance DC-link voltages. IEEE Transactions on Industrial Electronics 2002; 49(4):752-765.

19. Pan Z, Peng FZ, Corzine KA, Stefanovic VR, Leuthen JM, Gataric S. Voltage balancing control of diode-clamped multilevel rectifier/inverter systems. IEEE Transactions on Industry Applications 2005; 41(6):1698-1706. 
20. Marchesoni M, Vaccaro L. Operating limits in multilevel MPC inverters with active front ends. Proceedings of the International Symposium on Power Electronics, Electrical Drives, Automation and Motion SPEEDAM 2010, Pisa, Italy, 2010; 192-197.

21. Saeedifard M, Iravani R, Pou J. A space vector modulation approach for a back-to-back connected four-level converter. Proceedings of the Power Electronics Specialist Conference PESC 2007, Orlando, Florida, USA, 2007; 2043-2049.

22. Saeedifard M, Iravani R, Pou J. A space vector modulation strategy for a back-to-back five-level HVDC converter system. IEEE Transactions on Industrial Electronics 2009; 56(2);452-466.

23. P Kokotović P, HK Khalil, O’Reilly J. Singular perturbation methods in control. Analysis and design (SIAM edition). Society for Industrial and Applied Mathematics: Philadelphia, Pennsylvania, USA, 1999.

24. Francis BA. The linear multivariable regulator problem. SIAM Journal on Control and Optimization 1977; 15(3):486-505.

25. Francis BA, Wonham WM. The internal model principle for linear multivariable regulators. Applied Mathematics \& Optimization 1975; 2(2):170-194.

26. Francis BA, Wonham WM. The internal model principle of control theory. Automatica 1976; 12(5):457-465.

27. Isidori A, Byrnes CI. Output regulation of nonlinear systems. IEEE Transactions on Automatic Control 1990; 35(2):131-140.

28. Yang:12 Dong X, Zhao J. Output regulation for a class of switched nonlinear systems: an average dwell-time method. International Journal of Robust and Nonlinear Control 2013; 23(4):439-449.

29. Ping Z, Huang J. Global robust output regulation for a class of multivariable systems. International Journal of Robust and Nonlinear Control 2013;23(3):241-261.

30. Yang X, Huang J. New results on robust output regulation of nonlinear systems with a nonlinear exosystem. International Journal of Robust and Nonlinear Control 2012; 22(15):1703-1719.

31. Brown LJ, Zhang Q. Periodic disturbance cancellation with uncertain frequency. Automatica 2004; $40(4): 631-637$.

32. Landau ID, Alma M, Constantinescu A, Martinez JJ, Noë M. Adaptive regulation - Rejection of unknown multiple narrow band disturbances (a review on algorithms and applications). Control Engineering Practice 2011; 19(10):1168-1181.

33. Ding Z. Asymptotic rejection of general periodic disturbances in output-feedback nonlinear systems. IEEE Transactions on Automatic Control 2006; 51(2):303-308.

34. Bodson M. Rejection of periodic disturbances of unknown and time-varying frequency. International Journal of Adaptive Control and Signal Processing 2005; 19(2-3):67-88.

35. Pigg S, Bodson M. Adaptive harmonic steady-state disturbance rejection with frequency tracking. Asian Journal of Control 2013; 15(1):1-10.

36. Guo X, Bodson M. Adaptive rejection of disturbances having two sinusoidal components with close and unknown frequencies. Asian Journal of Control 2012; 14(1):36-44.

37. Sacks A, Bodson M, Khosla P. Experimental results of adaptive periodic disturbance cancellation in a high performance magnetic disk drive. ASME Journal of Dynamic Systems, Measurement, and Control 1996; 118:416perform.

38. Steinbuch M. Repetitive control for systems with uncertain period-time. Automatica 2002; 38(12):2103-2109.

39. Tsao TC, Qian YX, Nemani M. Repetitive control for asymptotic tracking of periodic signals with an unknown period. ASME Journal of Dynamic Systems, Measurement, and Control 2000; 122(2):364-369.

40. Ding Z. Global stabilization and disturbance suppression of a class of nonlinear systems with uncertain internal model. Automatica 2003; 39(3):471-479.

41. Umbría F, Vázquez S, Gordillo F, Gómez-Estern F. Observer-based direct power control for three-level NPC rectifiers. Proceedings of the 35th Annual Conference of IEEE Industrial Electronics IEEE-IECON 2009, Porto, Portugal, 2009; 1663-1668.

42. Umbría F, Gordillo F, Salas F, Vázquez S. Voltages balance control in three phase three-level NPC rectifiers. Proceedings of the IEEE International Symposium on Industrial Electronics ISIE 2010, Bari, Italy, 2010; 30183023.

43. Rodríguez J, Franquelo LG, Kouro S, León JI, Portillo RC, Prats MM, Pérez MA. Multilevel converters: an enabling technology for high-power applications. Proceedings of the IEEE 2009; 97(11):1786-1817.

44. Portillo RC, Prats MM, León JI, Sánchez JA, Carrasco JM, Galván E, Franquelo LG. Modeling strategy for backto-back three-level converters applied to high-power wind turbines. IEEE Transactions on Industrial Electronics 2006; 53(5):1483-1491.

45. F. Umbría, J. Aracil, and F. Gordillo. Singular perturbation stability analysis of three phase two-level power converters. In Proc. of the 18th Mediterranean Conference on Control and Automation, Marrakech, Morocco, 2010; $123-128$.

46. Vázquez S, Sánchez JA, Carrasco JM, León JI, Galván E. A model-based direct power control for three-phase power converters. IEEE Transactions on Industrial Electronics 2008; 55(4):1647-1657.

47. Chen D, Paden B. Nonlinear adaptive torque-ripple cancellation for step motors. Proceedings of the 29th IEEE Conference on Decision and Control IEEE-CDC 1990, Honolulu, USA, 1990; 3319-3324.

48. Chen D, Paden B. Adaptive linearization of hybrid step motors: stability analysis. IEEE Transactions on Automatic Control 1993; 38(6):874-887.

49. Bodson M, Douglas S. Adaptive algorithms for the rejection of sinusoidal disturbances with unknown frequency. Automatica 1997; 33(12):2213-2221.

50. Guo X, Bodson M. Analysis and implementation of an adaptive algorithm for the rejection of multiple sinusoidal disturbances. IEEE Transactions on Control Systems Technology 2009; 17(1):40-50. 